\title{
Impact of sarcopenia in borderline resectable and locally advanced pancreatic cancer patients receiving stereotactic body radiation therapy
}

\author{
William H. Jin ${ }^{1,2}$, Eric A. Mellon ${ }^{2}$, Jessica M. Frakes ${ }^{2}$, Gilbert Z. Murimwa, ${ }^{1,2}$, Pamela J. Hodul' ${ }^{2}$, Jose M. \\ Pimiento $^{2}$, Mokenge P. Malafa ${ }^{2}$, Sarah E. Hoffe ${ }^{2}$ \\ ${ }^{1}$ University of South Florida Morsani College of Medicine, Tampa, FL, USA; ${ }^{2}$ Moffitt Cancer Center, Tampa, FL, USA \\ Contributions: (I) Conception and design: SE Hoffe; (II) Administrative support: PJ Hodul, JM Pimiento, MP Malafa, SE Hoffe; (III) Provision of \\ study materials or patients: EA Mellon, PJ Hodul, JM Pimiento, MP Malafa, SE Hoffe; (IV) Collection and assembly of data: EA Mellon, WH Jin; (V) \\ Data analysis and interpretation: WH Jin; (VI) Manuscript writing: All authors; (VII) Final approval of manuscript: All authors. \\ Correspondence to: William H. Jin. 621 Island Place Way, Tampa, FL 33602, USA. Email: wjin@health.usf.edu.
}

Background: Total psoas area (TPA), a marker of sarcopenia, has been used as an independent predictor of clinical outcomes in gastrointestinal (GI) cancers as a proxy for frailty and nutritional status. Our study aimed to evaluate whether TPA, in contrast to traditional measurements of nutrition like body mass index (BMI) and body surface area (BSA), was predictive of outcomes in borderline resectable pancreatic cancer (BRPC) and locally advanced pancreatic cancer (LAPC) patients receiving stereotactic body radiation therapy (SBRT).

Methods: Retrospective analysis of an institutional review board approved database of 222 BRPC and LAPC treated with SBRT from 2009-2016 yielded 183 patients that met our selection criteria of preSBRT computed tomography (CT) imaging with an identifiable L4 vertebra. Once the L4 vertebral level was identified, the bilateral psoas muscles were manually contoured. This area was normalized by patient height, with units described in $\mathrm{mm}^{2} / \mathrm{m}^{2}$. Receiver operating characteristic (ROC) curves were generated for TPA, BMI, and BSA to elicit clinically relevant cutoffs. Regression and Kaplan-Meier analyses were used to correlate toxicity with survival functions.

Results: Low TPA (OR $=1.903, \mathrm{P}=0.036)$ was predictive of acute toxicities, and only TPA was predictive of Grade 3 or higher acute toxicities $(\mathrm{OR}=10.24, \mathrm{P}=0.007)$. Both findings were independent of tumor resectability. $\mathrm{Pain}(\mathrm{P}=0.003)$, fatigue $(\mathrm{P}=0.040)$, and nausea $(\mathrm{P}=0.039)$ were significantly associated with low TPA. No association was identified between any measurement of nutritional status and the development of late toxicities, overall survival, local progression or local recurrence. However, BRPC patients survived longer (median $=21.98$ months) than their LAPC (median $=16.2$ months) counterparts $(\mathrm{P}=0.002)$, independent of nutritional status.

Conclusions: TPA measurement is readily available and more specific than BMI or BSA as a predictor of acute radiotoxic complications following SBRT in BRPC/LAPC patients. A TPA of $<500 \mathrm{~mm}^{2} / \mathrm{m}^{2}$ is a clinically relevant cutoff that can direct physicians to address expected complications of pain, fatigue, and nausea. However, tumor resectability remains as the only predictor of overall survival in this cohort.

Keywords: Pancreatic cancer; cachexia; sarcopenia; radiation toxicity; computed tomography (CT)

Submitted Aug 02, 2017. Accepted for publication Sep 22, 2017.

doi: 10.21037/jgo.2017.09.13

View this article at: http://dx.doi.org/10.21037/jgo.2017.09.13 


\section{Introduction}

Pancreatic cancer is the $7^{\text {th }}$ leading cause of cancer death amongst adults in the US and is associated with 6\% 5-year overall survival rate for Stage II cancers (1), declining to $3 \%$ for patients with Stage III disease. Surgical resection offers the highest chance for remission, with 5-year survival rates of up to $20 \%(2-5)$. Unfortunately, many patients present with disease that is not amenable to potentially curative upfront resection (1). After neoadjuvant therapy, some borderline resectable (BRPC) and locally advanced pancreatic cancers (LAPC) may ultimately undergo a margin negative (R0) resection.

Markers of nutritional status have been predictive of outcomes in gastrointestinal cancers (6-8). Specifically, body mass index (BMI) has been widely evaluated due to its ability to normalize for patient height (9-14). Another metric, body surface area (BSA), has been incorporated clinically to personalize chemotherapeutic doses based on patient size and visceral fat content (15-17). A relatively new metric, total psoas area (TPA), has shown promise as an additional proxy for nutritional status; specifically, low TPA, or sarcopenia, has been used in place of deficient nutritional status in gastrointestinal cancers $(18,19)$.

Previous studies investigated the association between pre-operative and post-operative TPA with outcomes in patients with pancreatic cancer (19-25). However, none have examined TPA prior to neoadjuvant therapy. Furthermore, none have focused on morbidity and mortality outcomes of neoadjuvant radiation therapy, whether standard dose or high dose per fraction, in patients treated for pancreatic cancer.

The purpose of this study was to evaluate whether pre-treatment TPA, in contrast to traditional measures of nutritional status, like BMI and BSA, correlates with outcomes in BRPC and LAPC patients receiving stereotactic body radiation therapy (SBRT).

\section{Methods}

\section{Patient population}

After obtaining institutional review board (IRB) approval, a retrospective review of our 222 SBRT pancreatic cancer database yielded 183 patients that met criteria for selection in this study. We included 146 BRPC and 57 LAPC, per National Comprehensive Cancer Network (NCCN) criteria. Patient demographic details are summarized in
Table 1. Treatment details have been described in our earlier publications (26). In brief, patients were treated per our institution's pancreatic cancer clinical pathway, which included the initial staging studies of endoscopic ultrasound (EUS), pancreas protocol computed tomography (CT), and positron emission tomography (PET)/CT scan. Most patients received initial gemcitabine-based chemotherapy for 2-3 months followed by a five-fraction dose painted regimen of SBRT, with median of 40 Gy delivered to the region of tumor/vessel abutment. Those receiving conventional fractionation, those with upfront resectable disease or metastatic disease were excluded from this study. Additionally, only patients with lumbar CTs with clearly visible L4 lumbar vertebrae were included.

\section{Nutritional status determination}

TPA was calculated by using the pre-treatment planning axial lumbar CTs. We identified the first axial slice of the L4 vertebra after the L3-L4 interspace and contoured the area of the psoas muscle bilaterally using the Pinnacle Treatment Planning System (v9.2-9.8, Philips, Amsterdam, Netherlands). This cross-sectional volume was divided by the thickness of the axial slice to get a cross-sectional area. To normalize for body size, the combined bilateral psoas cross-sectional areas were divided by the height of the patient $(27,28)$.

$$
\operatorname{TPA}\left(\frac{\mathrm{mm}^{2}}{\mathrm{~m}^{2}}\right)=\frac{\text { Bilateral paoas area }\left(\mathrm{mm}^{2}\right)}{\text { Patient height }\left(\mathrm{m}^{2}\right)}
$$

BMI calculations were performed using the following equation.

$$
\operatorname{BMI}\left(\frac{\mathrm{kg}}{\mathrm{m}^{2}}\right)=\frac{\text { Patient } \text { weight }(\mathrm{kg})}{\text { Patient height }\left(\mathrm{m}^{2}\right)}
$$

BSA calculations were performed using the Mosteller formula (29).

$$
\text { BSA }=0.007184 \times \text { Patient weight }(\mathrm{kg})^{0.425} \times \text { Patient height }(\mathrm{m})^{0.725}
$$

\section{Acute and late radiation toxicities}

Acute radiation toxicities were evaluated once during the 5 -day course of SBRT by the treating radiation oncologist and subsequently 4-6 weeks after completion when the patient returned for re-staging evaluation or more frequently as needed. Acute radiation toxicities were defined 
per CTCAE version 4.0 (30-32). Patients were routinely followed in clinic after treatment, and late radiation toxicities were defined as symptoms that occurred 3 months or later post-treatment.

\section{Statistical analysis}

Patient characteristics were analyzed between sarcopenic and non-sarcopenic groups, high and low BMI, and high and low BSA with Pearson Chi-square tests. We used the median TPA in this dataset as a binary cutoff for statistical analysis of survival functions, as is common in the current literature $(23,25,33)$. Further, receiver operating characteristic (ROC) curves were also generated to determine a more sensitive and specific cutoff rather than using arbitrary median values and to validate literaturebased values for sarcopenia, BMI, and BSA when examining acute toxicities. Significant ROC binary cutoffs were further evaluated using binomial logistic regression. For multiple variables that were significant, multivariate regression was performed. Sub-group analysis was performed for both gender and patients of advanced age (AA, >75 years old). Statistical significance was set at $\mathrm{P}<0.05$. Overall survival, local progression and local recurrence were evaluated using Kaplan-Meier survival functions. Significance was evaluated with Mantel-Cox log ranks. Significant predictors of survival functions were then further analyzed using multivariate Cox regression. All statistical analysis and figure artwork was performed and generated using SPSS v 23.0.0.2 (IBM, Armonk, NY, USA).

\section{Results}

\section{Patient demographics}

Patient characteristics were compared by their sarcopenic status (binary cutoff at median TPA of 533), median BMI (24.5), and median BSA (1.79). We found that using a binary cutoff at the median age [66] was not a significant predictor of TPA. While this specific age cutoff did not correlate with BMI, patients of AA were 2.8 times more likely to be sarcopenic $(\mathrm{P}=0.008)$ and were 2.9 times more likely to have a lower BSA $(\mathrm{P}=0.008)$ than their younger counterparts. $36.2 \%$ of patients younger than 75 were sarcopenic, with a median TPA of 553 , while the majority $68.1 \%$ of patients older than 75 were sarcopenic, with a median TPA of 458 .

Women were found to have significantly lower TPA
$(\mathrm{P}<0.001)$ and BSA $(\mathrm{P}<0.001)$ than men, but $\mathrm{BMI}$ was not correlated with gender (Table 1). With a median TPA of 466 and 585 , respectively, $58.1 \%$ of women and $25.1 \%$ of men were sarcopenic. Most tumors [152] were in the head of the pancreas, with only 30 in the body and 1 in the tail. This cohort consisted entirely of borderline resectable or locally advanced tumors. When stratified by BSA, there were a significantly higher number of patients with stage $\mathrm{T} 4$ cancers in the lower BSA group $(\mathrm{P}=0.01)$. In addition, patients with low BSA were also more likely to be of a higher AJCC stage $(\mathrm{P}=0.018)$ and locally advanced $(\mathrm{P}=0.008)$. This significance was not found in either the TPA or BMI groups.

\section{Acute and late radiation toxicities}

We generated binary cutoffs with ROC curves to obtain a cohort-specific value for acute toxicities. Our ROC curve predicted that a cutoff of 497 would give the highest combination of sensitivity (49.5\%) and specificity (75\%) in predicting radiation toxicities ( $\mathrm{AUC}=0.590, \mathrm{P}=0.040$ ). Therefore, for analyses of radiation toxicities, we defined sarcopenia as a TPA $<497$. Based on this cutoff, our binomial logistic regression model predicted that patients with a low TPA had a 2.55 higher likelihood of developing acute radiation toxicities $(\mathrm{P}=0.004)$. Sub-group analysis in patients of AA did not show increased radiotoxicities with either a high or low TPA. Rather, only patients younger than 75 with a low TPA were 2.7 times more likely to develop acute radiotherapy complications (Table 2, $\mathrm{P}=0.002)$. Sub-group analysis with gender indicated that in males, TPA was not a significant predictor of complications. However, females with a low TPA were 3.8 times more likely than females with a high TPA to develop complications (Table 3, $\mathrm{P}=0.004$ ).

ROC curves generated for BMI were not statistically significant. We continued to use the median BMI as a cutoff for high and low BMI when analyzing acute radiation toxicities. ROC curves for BSA maximized the sensitivity (62.2\%) and specificity (52.8\%) at a binary cutoff of 1.84 (AUC $=0.598, \mathrm{P}=0.025)$. Subsequently, our regression model using this cutoff predicted a 1.84 higher likelihood of developing acute radiation toxicities in patients with a lower BSA $(\mathrm{P}=0.048)$. Therefore, for analyses of radiation toxicities, we defined low BSA as $<1.84$.

We compared our cohort-specific ROC-derived definition of sarcopenia to the median cutoff and found 
Table 1 Patient characteristics

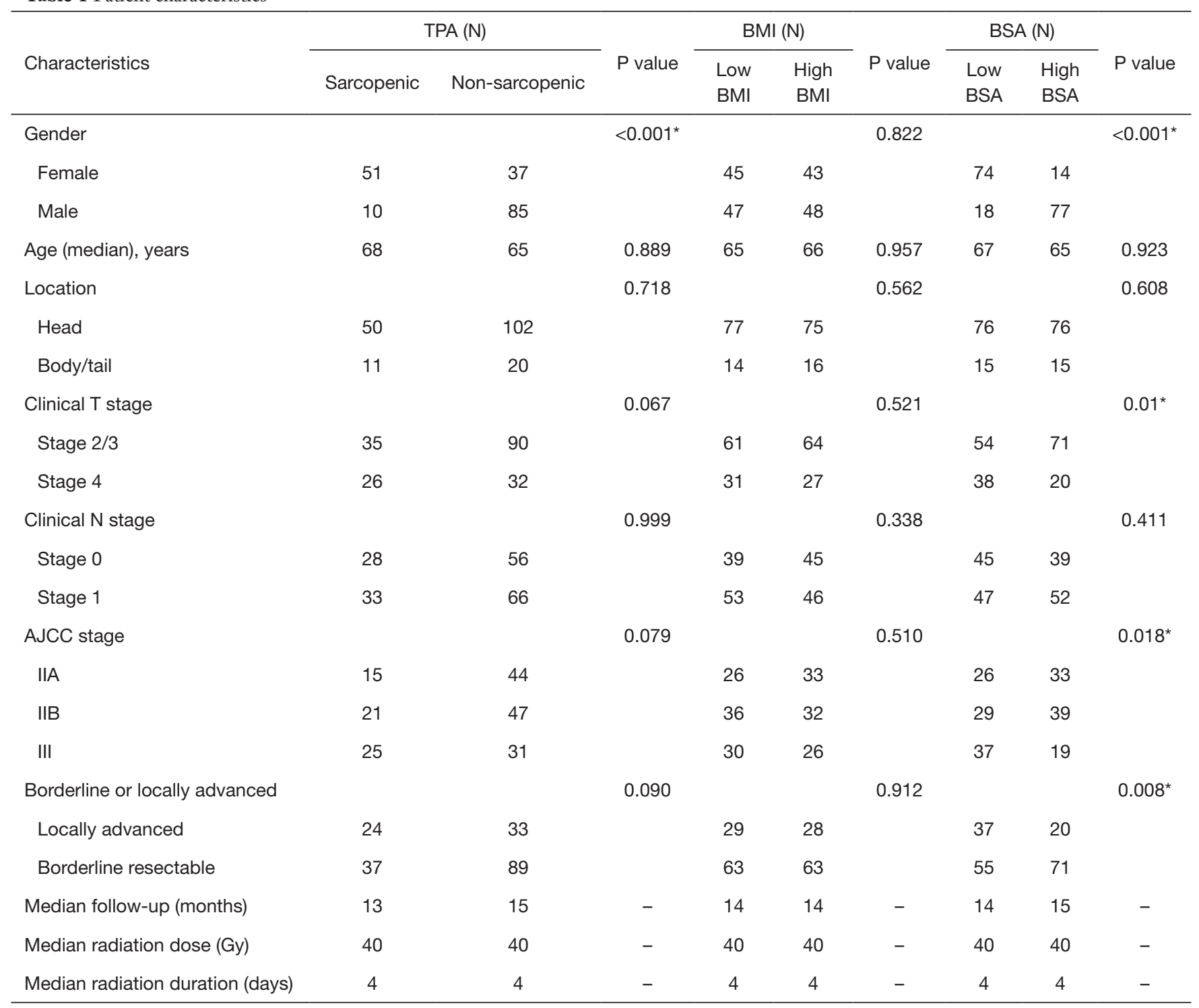

${ }^{*}$, denotes significance $<0.05$. TPA, total psoas area; BMI, body mass index; BSA, body surface area.

Table 2 Sarcopenic stratification of patients based on advanced age status

\begin{tabular}{llc}
\hline \multirow{2}{*}{ Age status } & \multicolumn{2}{c}{ Acute radiation toxicity, N [\%] } \\
\cline { 2 - 3 } & None & Any grade \\
\hline Age $<75$ years & & \\
Sarcopenic & $14[26]$ & $40[74]$ \\
Non-sarcopenic & $46[48]$ & $49[52]$ \\
Age $>75$ years & & \\
Sarcopenic & $6[29]$ & $15[71]$ \\
Non-sarcopenic & $6[46]$ & $7[53]$ \\
\hline
\end{tabular}

Table 3 Sarcopenic stratification of patients based on gender

\begin{tabular}{lcc}
\hline \multirow{2}{*}{ Gender } & \multicolumn{2}{c}{ Acute rad toxicity, N [\%] } \\
\cline { 2 - 3 } & None & Any grade \\
\hline Female & $11[22]$ & $40[78]$ \\
Sarcopenic & $19[51]$ & $18[49]$ \\
Non-sarcopenic & & \\
Male & $9[35]$ & $15[65]$ \\
Sarcopenic & $33[46]$ & $38[54]$ \\
Non-sarcopenic & & \\
\hline
\end{tabular}


Table 4 Distribution of acute radiation toxicities by nutritional proxy

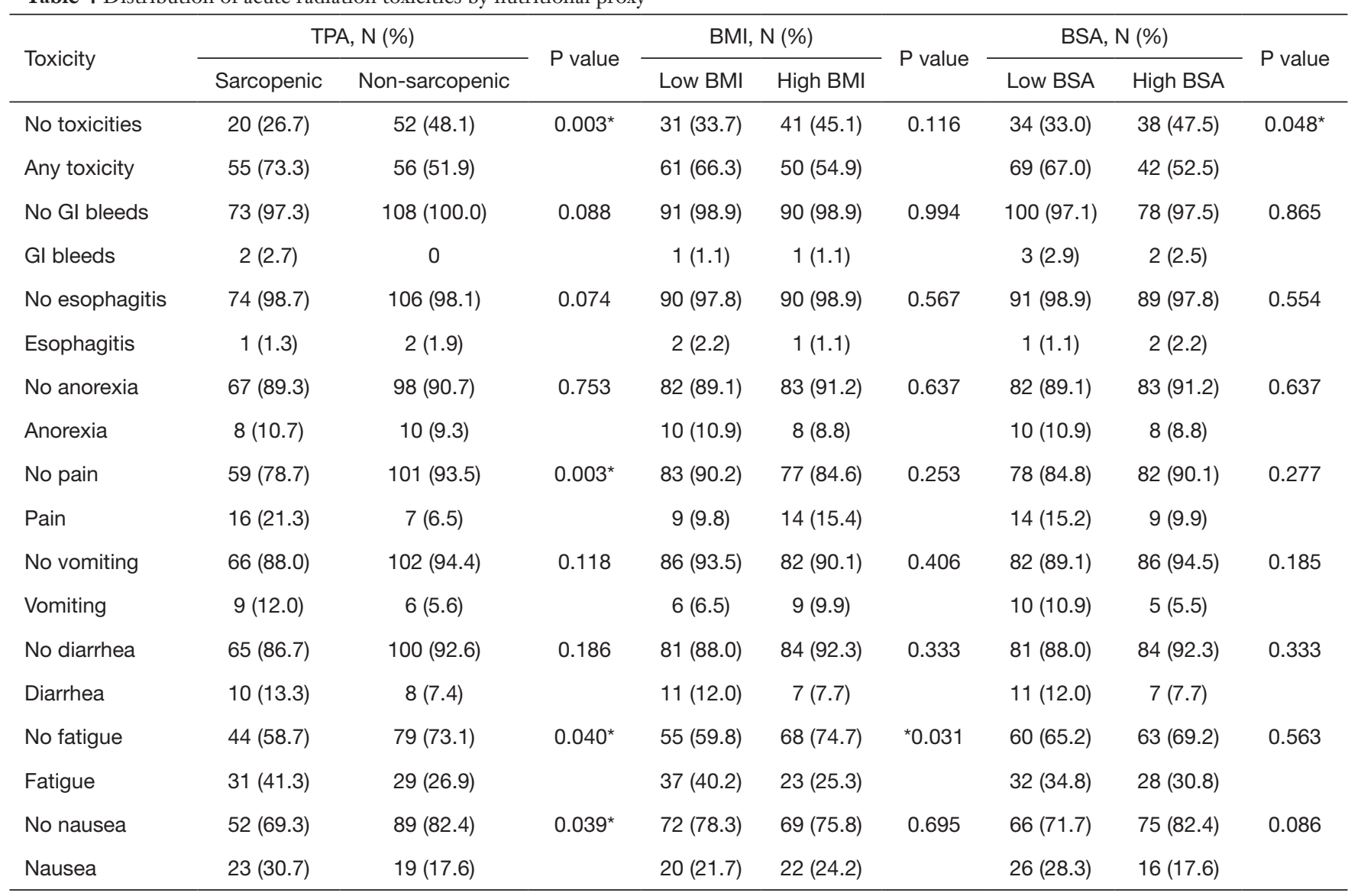

${ }^{*}$, denotes significance $<0.05$. TPA, total psoas area; BMI, body mass index; BSA, body surface area.

similar results where non-sarcopenic patients were twice as likely to report no acute radiation toxicities $(\mathrm{P}=0.019)$. Therefore, we elected to use the ROC-derived sarcopenic cutoff due to its increased specificity. BMI was not able to predict radiation toxicities, but BSA was $(\mathrm{P}=0.048)$. In addition, sarcopenic patients were significantly more likely to develop grade 3 or higher acute radiation toxicities than non-sarcopenic patients $(\mathrm{P}=0.007)$.

In the sarcopenic group, patients experienced significantly more acute radiation toxicities, of any grade, than their non-sarcopenic counterparts $(\mathrm{P}=0.003) .73 .3 \%$ of sarcopenic patients experienced some form of radiation toxicity, compared to $51.9 \%$ of non-sarcopenic patients (Table 4). Specifically, they experienced more pain $(21.3 \%$ vs. $6.5 \%, \mathrm{P}=0.003)$, fatigue ( $41.3 \%$ vs. $26.9 \%, \mathrm{P}=0.040)$, and nausea $(30.7 \%$ vs. $17.6 \%, \mathrm{P}=0.039)$. Specific breakdowns of individually graded complications are listed in Table S1. There were no significant differences in TPA, BMI, or BSA in developing late onset radiation toxicities.

\section{Survival functions}

Even with gender or age stratification, no proxy for nutritional status was a significant predictor of overall survival, local progression, or local recurrence. However, patients presenting with LAPC were associated with worse overall survival than BRPC patients $(\mathrm{P}=0.002)$. They had a median survival of 14.1 months, while their BRPC counterparts had a median survival of 18.4 months. Similarly, clinical AJCC Stage was predictive of overall survival $(\mathrm{P}=0.005)$. Stage III patients had a median survival of 14.1 months, while Stage IIB had a median survival of 16.6 months and Stage IIA patients had a median survival of 21.1 months. Stage II (IIA and IIB) patients, as a cohort, had a median survival of 18.4 months. When compared to Stage III patients, they survived significantly longer 
$(\mathrm{P}=0.001)$. Due to the high collinearity of AJCC stage and upfront resectability, multivariate Cox regression was not performed.

\section{Discussion}

In our study, we used pre-treatment planning CTs to evaluate the association between skeletal muscle radiographic features and acute complications in patients with BRPC and LAPC. While multiple studies have attempted to validate a clinically relevant TPA cutoff, the difficulty lies in producing large scale studies with a population relevant value. The next best option is to use data from multiple studies using percentile based summative values. The literature currently uses a plethora of cohort-specific values, including using the lowest median, tertile, quartile, quintile, or standard deviation (19-21,23,24,27,28,33-38) in the study cohort. We validated our cohort's cutoff, by performing ROC curve analysis with regards to acute radiotoxicities which correlates to approximately a TPA of 500. Unfortunately, time-dependent ROC curves have yet to be a viable and reproducible method of creating a binary cutoff for survival functions (39). Ideally, a programmable function in a commercially available statistics program would encompass a time-dependent ROC function since Kaplan-Meier curves are used so commonly in clinical research. Currently, the best method involves using either cohort-specific cutoffs or arbitrary cutoffs validated in the literature.

TPA and BSA were found to be significantly lower in elderly females in this study and subsequent calculations were performed using sub-group analysis controlling for gender and age. Our findings echo the current literature, as young, male patients were found to have higher muscle mass than elderly female patients $(21,24,29)$. Previous authors have identified age-dependent, or primary, sarcopenia as a confounder in studies focused on sarcopenia secondary to cancer, like ours. Age-dependent sarcopenia had to be effectively isolated as a confounder since it is highly prevalent in the elderly population, specifically in patients of AA (21). Interestingly, our cohort had 34 patients over the age of 75 , yet despite a higher rate of sarcopenia in this group, TPA was not a significant predictor of acute complications. One interpretation and explanation reinforces the concept that patients with sarcopenia secondary to cachexic processes are more likely to develop acute radiotoxic complications. Otherwise, TPA should have continued to be predictive of complications as age increases.
In patients younger than 75 , there was a $36.2 \%$ incidence of sarcopenia. In this group, sarcopenic patients were 2.68 times more likely to develop acute radiotoxic complications than their counterparts $(\mathrm{P}=0.008)$. In contrast, there was a $61.8 \%$ incidence of sarcopenia in patients of AA. The nearly two-fold increase in the incidence of sarcopenia in our elderly cohort can be attributed to either the emergence of primary sarcopenia or the development of secondary sarcopenia. We are limited in determining a definitive etiology in these patients given the retrospective nature of the study and can only hypothesize, as we could not measure serum cytokine levels or assess each patient's inflammatory state. However, we expect a lower proportion of age-dependent sarcopenia and a higher proportion of sarcopenia secondary to a pathologic process of malignancy in our younger patients. For the clinician, the specificity of sarcopenia status decreases significantly in the elderly, but that should not downplay its sensitivity in detecting which patients may need additional interventions.

Additional sub-group analysis with gender showed that sarcopenic females were almost 4 times more likely to develop acute complications than non-sarcopenic females. Conversely, sarcopenic males were not nearly so affected. This gender specific attribute may provide additional insight into the pathophysiological process of secondary sarcopenia. Current research suggests that a proinflammatory state is mediated by release of cytokines like IL-6 (40-44). Well-known for their anabolic effects and utility in other muscle wasting states like HIV, androgens may also contribute to the gender differences seen in TPAs via multiple mechanisms (45-49). Postmenopausal females may be particularly predisposed to sarcopenia due to the absence of the anabolic effects of circulating testosterone in addition to losing the anti-inflammatory effects of estrogen. Estrogen has been shown to have anti-inflammatory properties and men continue to aromatize estrogen so long as they have circulating testosterone (50-59). In our study, we did not have data on whether our post-menopausal patients were on estrogen replacement therapy (ERT) and future studies are needed to compare the rate of sarcopenia and complications in this patient group. However, our findings suggest that clinicians should pay special attention to women with low muscle mass, as they may require earlier intervention than other patients. In this study, we were unable to assess baseline TPA prior to systemic chemotherapy administration, thus we cannot conclude if there was a significant change prior to the time point of our study which was defined as the TPA measured from 
the SBRT treatment planning CT after the completion of neoadjuvant systemic therapy.

TPA was significantly higher in young males, as was expected due to the gender and age difference $(21,24)$. However, even after sub-group analysis was performed, neither TPA, BMI, nor BSA were significant predictors of overall survival. While TPA and BSA could significantly predict the development of acute radiation complications, BMI could not. BMI has been found to be a useful metric in outcomes related to obesity, and rarely has it found usefulness for low BMI $(24,60)$. When dealing with a patient population that is generally underweight, we found no significant role for BMI regarding our selected outcomes.

Current best practice guidelines indicate that BRPC and LAPC can be managed with initial neoadjuvant therapy, as surgical resection remains the highest chance of survival for the patient (61-63). While our study did not find a significant association between proxies of nutritional status prior to neoadjuvant SBRT therapy and mortality, we found that it was associated with significant morbidity. As neoadjuvant therapy regimens containing SBRT will likely continue to be a component in BRPC/LAPC treatment pending completion of ongoing trials, the timely management of morbidities associated with radiation therapy remain important to optimize the patient's status prior to potential surgery.

In the last decade, pancreatic SBRT has evolved significantly since the first single-fraction phase I dose escalation studies. From the Stanford study, which reported no patients with grade 3 or higher acute toxicity (64) and the 45 Gy in 3-fraction Danish study reporting acute toxicity of $79 \%$ grade 2 and $4.5 \%$ grade 4 (65), review of the various techniques have indeed shown the importance of dosimetric and treatment planning factors to limit acute morbidity, suggesting that multiple fraction regimens, smaller target volumes, and accounting for respiratory-associated tumor motion can decrease toxicity (66). Integrating these advances, a recent prospective, multi-institutional trial for patients with LAPC utilizing a 5 -fraction regimen of 33 Gy reported rates of grade $\geq 2$ gastritis, fistula, enteritis, and ulcer to be $2 \%$ (67). Furthermore, rates of resection among BRPC and LAPC patients are increasing, with $21.6 \%$ of patients (79\% of whom were LAPC) in a series from Johns Hopkins undergoing resection and $51 \%$ of BRPC/ $24 \%$ of LAPC treated at Moffitt with induction FOLFIRINOX (leucovorin, fluorouracil, irinotecan, oxaliplatin) undergoing surgery $(26,68)$. Integration of more active systemic therapies such as mFOLFIRINOX (neoadjuvant modified FOLFIRINOX) with SBRT is now being tested, with early data from a phase I trial using a strategy of 30-36 Gy in 3 fractions to the whole target and a focal 9 Gy integrated boost to the tumor/vessel interface showing no acute grade 3 or 4 complications and a median survival not yet reached (69). As the investigation into the optimal neoadjuvant systemic therapy agents, SBRT dosing, and treatment sequencing continues, more emphasis will continue to be placed on preparing patients for surgery to achieve an $\mathrm{R} 0$ resection.

Outcome results in this analysis indicate that the median difference in survival in BRPC and LAPC patients is separated by a mere six months, like other findings in the current literature $(35,70,71)$. Our study specifically included patients with only borderline resectable and locally advanced disease, which correlates with IIA, IIB, and III AJCC stages. In our results, having Stage III disease was the most significant predictor of overall survival. This also concurs with the current literature that even in patients with clinically evident cachexia, secondary sarcopenia, or decreased visceral fat adiposity, survival is contingent on whether they proceed to resection $(21,72-75)$, which is uncertain in these BRPC and LAPC patient populations.

The utility of TPA finds its niche in predicting which patients might develop short-term radiation complications. Clinicians should be cautious to evaluate female patients with a low TPA since they are more likely to need additional nutritional supplementation, pain control or anti-emetics during their neoadjuvant treatment to avoid discontinuation of therapy due to complications. Future research should evaluate the effectiveness of strategies to incorporate such supportive measures as part of the preoperative conditioning regimen. With neoadjuvant intent to facilitate margin negative resection, avoiding grade 3 or higher acute complications is important to prevent unnecessary delay of attempted surgery. In addition to optimizing nutrition, future work is needed to determine whether adding a strength training regimen to the 3-6 months period leading up to potential surgery in sarcopenic patients could improve outcomes.

Knowing whether low muscle mass, from a single snapshot in time, or a loss of muscle mass, viewed as a longitudinal process, predicts acute radiotoxic complications better remains to be seen, as we were limited by the retrospective nature of this study. However, our results indicate that sarcopenia at the time of SBRT planning is a strong predictor for acute complications after SBRT. Previous studies have shown that weight loss could be a 
more reliable metric of frailty than radiographic measures and should also be examined in future studies (21).

Additionally, Amini et al. found that perioperative total psoas volume was a more accurate predictor of morbidity and mortality following pancreaticoduodenectomy, specifically post-operative complications and overall survival (20). This questions the utility of a single axial slice and its trade-off with prognostic efficiency for accuracy and future analysis can determine if this significant difference is seen in neoadjuvant therapy as well. If possible, total psoas volume in comparison to TPA as a metric for sarcopenia should be explored too. Finally, while evaluation of chemotherapy complications in the months preceding SBRT was outside the scope of this project, it should be integrated in future analyses to provide a composite of the totality of neoadjuvant patient factors.

\section{Conclusions}

Pre-neoadjuvant SBRT sarcopenic status was not found to be a significant predictor of overall survival in BRPC or LAPC patients. The most useful predictors of survival in pancreatic cancer patients receiving neoadjuvant therapy prior to surgery are still tumor resectability or clinical AJCC stage. However, we found that sarcopenia is a robust proxy for deficient nutritional status and the development of high grade acute radiation toxicities. Even more so in females, these complications can and should be managed by a well-trained clinician to improve the likelihood of such patients maintaining fitness for attempted surgical resection without delay. Using a TPA of $500 \mathrm{~mm}^{2} / \mathrm{m}^{2}$, as a proxy for sarcopenia, is a reasonable, non-arbitrary cutoff for predicting which borderline resectable or LAPC patients may need more aggressive nutritional supplementation, anti-emetics, or appetite stimulants during their neoadjuvant treatment. This study is the first to examine patient specific factors immediately preceding SBRT with the intention of potential enhancement of margin negative resection. Future studies are needed to determine which conditioning regimens may be best to optimize sarcopenic patients for eventual surgery and whether more conservative constraints for the GI mucosa adjacent to the tumor target in this patient group may be necessary to further decrease acute grade $3+$ complications.

\section{Acknowledgements}

None.

\section{Footnote}

Conflicts of Interest: The authors have no conflicts of interest to declare.

Ethical Statement: The study was approved by institutional review board of University of South Florida (No. \# Pro00003382) and informed consent was taken from all the patients.

\section{References}

1. Ilic M, Ilic I. Epidemiology of pancreatic cancer. World J Gastroenterol 2016;22:9694-705.

2. Cameron JL, Crist DW, Sitzmann JV, et al. Factors influencing survival after pancreaticoduodenectomy for pancreatic cancer. Am J Surg 1991;161:120-4; discussion 124-5.

3. Epelboym I, DiNorcia J, Winner M, et al. Neoadjuvant therapy and vascular resection during pancreaticoduodenectomy: shifting the survival curve for patients with locally advanced pancreatic cancer. World J Surg 2014;38:1184-95.

4. Stephens J, Kuhn J, O'Brien J, et al. Surgical morbidity, mortality, and long-term survival in patients with peripancreatic cancer following pancreaticoduodenectomy. Am J Surg 1997;174:600-3; discussion 603-4.

5. Yeh CC, Jeng YM, Ho CM, et al. Survival after pancreaticoduodenectomy for ampullary cancer is not affected by age. World J Surg 2010;34:2945-52.

6. Ihira H, Mizumoto A, Makino K, et al. Physical functions, health-related outcomes, nutritional status, and blood markers in community-dwelling cancer survivors aged 75 years and older. Asian Pac J Cancer Prev 2014;15:3305-10.

7. Potischman N, Hoover RN, Brinton LA, et al. The relations between cervical cancer and serological markers of nutritional status. Nutr Cancer 1994;21:193-201.

8. Tan CS, Read JA, Phan VH, et al. The relationship between nutritional status, inflammatory markers and survival in patients with advanced cancer: a prospective cohort study. Support Care Cancer 2015;23:385-91.

9. Li D, Morris JS, Liu J, et al. Body mass index and risk, age of onset, and survival in patients with pancreatic cancer. JAMA 2009;301:2553-62.

10. Shi YQ, Yang J, Du P, et al. Effect of Body Mass Index on Overall Survival of Pancreatic Cancer: A Meta-Analysis. Medicine (Baltimore) 2016;95:e3305.

11. Choi Y, Kim TY, Lee KH, et al. The impact of body mass index dynamics on survival of patients with advanced 
pancreatic cancer receiving chemotherapy. J Pain Symptom Manage 2014;48:13-25.

12. Yuan C, Bao Y, Wu C, et al. Prediagnostic body mass index and pancreatic cancer survival. J Clin Oncol 2013;31:4229-34.

13. Dalal S, Hui D, Bidaut L, et al. Relationships among body mass index, longitudinal body composition alterations, and survival in patients with locally advanced pancreatic cancer receiving chemoradiation: a pilot study. J Pain Symptom Manage 2012;44:181-91.

14. Pelucchi C, Galeone C, Polesel J, et al. Smoking and body mass index and survival in pancreatic cancer patients. Pancreas 2014;43:47-52.

15. Kaestner SA, Sewell GJ. Chemotherapy dosing part I: scientific basis for current practice and use of body surface area. Clin Oncol (R Coll Radiol) 2007;19:23-37.

16. Hunter RJ, Navo MA, Thaker PH, et al. Dosing chemotherapy in obese patients: actual versus assigned body surface area (BSA). Cancer Treat Rev 2009;35:69-78.

17. Pinkel D. The use of body surface area as a criterion of drug dosage in cancer chemotherapy. Cancer Res 1958;18:853-6.

18. Ozola Zalite I, Zykus R, Francisco Gonzalez M, et al. Influence of cachexia and sarcopenia on survival in pancreatic ductal adenocarcinoma: a systematic review. Pancreatology 2015;15:19-24.

19. Onesti JK, Wright GP, Kenning SE, et al. Sarcopenia and survival in patients undergoing pancreatic resection. Pancreatology 2016;16:284-9.

20. Amini N, Spolverato G, Gupta R, et al. Impact Total Psoas Volume on Short- and Long-Term Outcomes in Patients Undergoing Curative Resection for Pancreatic Adenocarcinoma: a New Tool to Assess Sarcopenia. J Gastrointest Surg 2015;19:1593-602.

21. Carrara G, Pecorelli N, De Cobelli F, et al. Preoperative sarcopenia determinants in pancreatic cancer patients. Clin Nutr 2017;36:1649-53.

22. Makiura D, Ono R, Inoue J, et al. Preoperative sarcopenia is a predictor of postoperative pulmonary complications in esophageal cancer following esophagectomy: A retrospective cohort study. J Geriatr Oncol 2016;7:430-6.

23. Underwood PW, Cron DC, Terjimanian MN, et al. Sarcopenia and failure to rescue following liver transplantation. Clin Transplant 2015;29:1076-80.

24. Mei KL, Batsis JA, Mills JB, et al. Sarcopenia and sarcopenic obesity: do they predict inferior oncologic outcomes after gastrointestinal cancer surgery? Perioper Med (Lond) 2016;5:30.

25. Otsuji H, Yokoyama Y, Ebata T, et al. Surgery-Related
Muscle Loss and Its Association with Postoperative Complications After Major Hepatectomy with Extrahepatic Bile Duct Resection. World J Surg 2017;41:498-507.

26. Mellon EA, Hoffe SE, Springett GM, et al. Long-term outcomes of induction chemotherapy and neoadjuvant stereotactic body radiotherapy for borderline resectable and locally advanced pancreatic adenocarcinoma. Acta Oncol 2015;54:979-85.

27. Shen W, Punyanitya M, Wang Z, et al. Total body skeletal muscle and adipose tissue volumes: estimation from a single abdominal cross-sectional image. J Appl Physiol (1985) 2004;97:2333-8.

28. Mourtzakis M, Prado CM, Lieffers JR, et al. A practical and precise approach to quantification of body composition in cancer patients using computed tomography images acquired during routine care. Appl Physiol Nutr Metab 2008;33:997-1006.

29. Mosteller RD. Simplified calculation of body-surface area. N Engl J Med 1987;317:1098.

30. Basch E, Reeve BB, Mitchell SA, et al. Development of the National Cancer Institute's patient-reported outcomes version of the common terminology criteria for adverse events (PRO-CTCAE). J Natl Cancer Inst 2014;106.

31. Kluetz PG, Chingos DT, Basch EM, et al. PatientReported Outcomes in Cancer Clinical Trials: Measuring Symptomatic Adverse Events With the National Cancer Institute's Patient-Reported Outcomes Version of the Common Terminology Criteria for Adverse Events (PROCTCAE). Am Soc Clin Oncol Educ Book 2016;35:67-73.

32. Dueck AC, Mendoza TR, Mitchell SA, et al. Validity and Reliability of the US National Cancer Institute's PatientReported Outcomes Version of the Common Terminology Criteria for Adverse Events (PRO-CTCAE). JAMA Oncol 2015;1:1051-9.

33. Krell RW, Kaul DR, Martin AR, et al. Association between sarcopenia and the risk of serious infection among adults undergoing liver transplantation. Liver Transpl 2013;19:1396-402.

34. Zakaria HM, Basheer A, Boyce-Fappiano D, et al. Application of morphometric analysis to patients with lung cancer metastasis to the spine: a clinical study. Neurosurg Focus 2016;41:E12.

35. Bachmann J, Heiligensetzer M, Krakowski-Roosen H, et al. Cachexia worsens prognosis in patients with resectable pancreatic cancer. J Gastrointest Surg 2008;12:1193-201.

36. Nattenmueller J, Hoegenauer H, Boehm J, et al. CT-based compartmental quantification of adipose tissue versus body metrics in colorectal cancer patients. Eur Radiol 
2016;26:4131-40.

37. Park SY, Yoon JK, Lee SJ, et al. Prognostic value of preoperative total psoas muscle area on long-term outcome in surgically treated oesophageal cancer patients. Interact Cardiovasc Thorac Surg 2017;24:13-9.

38. Paknikar R, Friedman J, Cron D, et al. Psoas muscle size as a frailty measure for open and transcatheter aortic valve replacement. J Thorac Cardiovasc Surg 2016;151:745-51.

39. Heagerty PJ, Lumley T, Pepe MS. Time-dependent ROC curves for censored survival data and a diagnostic marker. Biometrics 2000;56:337-44.

40. Fearon KC, Voss AC, Hustead DS, et al. Definition of cancer cachexia: effect of weight loss, reduced food intake, and systemic inflammation on functional status and prognosis. Am J Clin Nutr 2006;83:1345-50.

41. Mitsunaga S, Ikeda M, Nakachi K, et al. Clinical significance of elevation of the serum IL-6 level in patients with advanced pancreatic cancer. J Clin Oncol 2011;29:181.

42. Schaap LA, Pluijm SM, Deeg DJ, et al. Inflammatory markers and loss of muscle mass (sarcopenia) and strength. Am J Med 2006;119:526 e9-17.

43. Leng S, Chaves P, Koenig K, et al. Serum interleukin-6 and hemoglobin as physiological correlates in the geriatric syndrome of frailty: a pilot study. J Am Geriatr Soc 2002;50:1268-71.

44. Fogelman DR, Wang XS, Hassan M, et al. Relative value of serum cytokines and clinical factors in predicting weight loss in advanced pancreatic cancer (PC). J Clin Oncol 2011;29:208.

45. Dardevet D, Remond D, Peyron MA, et al. Muscle wasting and resistance of muscle anabolism: the "anabolic threshold concept" for adapted nutritional strategies during sarcopenia. ScientificWorldJournal 2012;2012:269531.

46. Woerdeman J, de Ronde W. Therapeutic effects of anabolic androgenic steroids on chronic diseases associated with muscle wasting. Expert Opin Investig Drugs 2011;20:87-97.

47. Timmerman KL, Rasmussen BB. Does a reduction in anabolic signaling contribute to muscle wasting in chronic heart failure? J Appl Physiol (1985) 2011;110:869-70.

48. Burckart K, Beca S, Urban RJ, et al. Pathogenesis of muscle wasting in cancer cachexia: targeted anabolic and anticatabolic therapies. Curr Opin Clin Nutr Metab Care 2010;13:410-6.

49. Cuthbertson D, Smith K, Babraj J, et al. Anabolic signaling deficits underlie amino acid resistance of wasting, aging muscle. FASEB J 2005;19:422-4.
50. Zhang E, Zhang H, Liu F, et al. Estrogen exerts antiinflammatory effects by inhibiting NF-kappaB pathway through binding with estrogen receptor beta on synovicytes of osteoarthritis. Xi Bao Yu Fen Zi Mian Yi Xue Za Zhi 2016;32:1605-9.

51. Pelekanou V, Kampa M, Kiagiadaki F, et al. Estrogen antiinflammatory activity on human monocytes is mediated through cross-talk between estrogen receptor ERalpha36 and GPR30/GPER1. J Leukoc Biol 2016;99:333-47.

52. Xu L, Liu JT. The anti-inflammatory effect and mechanisms of estrogen in cardiocerebrovascular diseases. Sheng Li Ke Xue Jin Zhan 2008;39:155-8.

53. Rozenberg S, Twagirayezu P, Vyankadondera J, et al. Effect of estrogen replacement therapy with or without concomitant nonsteroidal anti-inflammatory drugs on pulsatility and resistance index of uterine arteries in healthy postmenopausal women. Int J Fertil Womens Med 1997;42:240-4.

54. Josefsson E, Tarkowski A, Carlsten H. Anti-inflammatory properties of estrogen. I. In vivo suppression of leukocyte production in bone marrow and redistribution of peripheral blood neutrophils. Cell Immunol 1992;142:67-78.

55. Spangler AS, Antoniades HN, Sotman SL, et al. Enhancement of the anti-inflammatory action of hydrocortisone by estrogen. J Clin Endocrinol Metab 1969;29:650-5.

56. Merlotti D, Gennari L, Stolakis K, et al. Aromatase activity and bone loss in men. J Osteoporos 2011;2011:230671.

57. Rochira V, Carani C. Aromatase deficiency in men: a clinical perspective. Nat Rev Endocrinol 2009;5:559-68.

58. de Ronde $W$. Therapeutic uses of aromatase inhibitors in men. Curr Opin Endocrinol Diabetes Obes 2007;14:235-40.

59. Gennari L, Nuti R, Bilezikian JP. Aromatase activity and bone homeostasis in men. J Clin Endocrinol Metab 2004;89:5898-907.

60. Tan BH, Birdsell LA, Martin L, et al. Sarcopenia in an overweight or obese patient is an adverse prognostic factor in pancreatic cancer. Clin Cancer Res 2009;15:6973-9.

61. Versteijne E, van Eijck CH, Punt CJ, et al. Preoperative radiochemotherapy versus immediate surgery for resectable and borderline resectable pancreatic cancer (PREOPANC trial): study protocol for a multicentre randomized controlled trial. Trials 2016;17:127.

62. Mahipal A, Frakes J, Hoffe S, et al. Management of borderline resectable pancreatic cancer. World J Gastrointest Oncol 2015;7:241-9.

63. Fathi A, Christians KK, George B, et al. Neoadjuvant therapy for localized pancreatic cancer: guiding principles. 
J Gastrointest Oncol 2015;6:418-29.

64. Koong AC, Le QT, Ho A, et al. Phase I study of stereotactic radiosurgery in patients with locally advanced pancreatic cancer. Int J Radiat Oncol Biol Phys 2004;58:1017-21.

65. Hoyer M, Roed H, Sengelov L, et al. Phase-II study on stereotactic radiotherapy of locally advanced pancreatic carcinoma. Radiother Oncol 2005;76:48-53.

66. Pollom EL, Alagappan M, von Eyben R, et al. Singleversus multifraction stereotactic body radiation therapy for pancreatic adenocarcinoma: outcomes and toxicity. Int J Radiat Oncol Biol Phys 2014;90:918-25.

67. Herman JM, Chang DT, Goodman KA, et al. Phase 2 multi-institutional trial evaluating gemcitabine and stereotactic body radiotherapy for patients with locally advanced unresectable pancreatic adenocarcinoma. Cancer 2015;121:1128-37.

68. Moningi S, Dholakia AS, Raman SP, et al. The Role of Stereotactic Body Radiation Therapy for Pancreatic Cancer: A Single-Institution Experience. Ann Surg Oncol 2015;22:2352-8.

69. Shaib WL, Hawk N, Cassidy RJ, et al. A Phase 1 Study of Stereotactic Body Radiation Therapy Dose Escalation for Borderline Resectable Pancreatic Cancer After Modified FOLFIRINOX (NCT01446458). Int J Radiat Oncol Biol

Cite this article as: Jin WH, Mellon EA, Frakes JM, Murimwa GZ, Hodul PJ, Pimiento JM, Malafa MP, Hoffe SE. Impact of sarcopenia in borderline resectable and locally advanced pancreatic cancer patients receiving stereotactic body radiation therapy. J Gastrointest Oncol 2018;9(1):24-34. doi: 10.21037/ jgo.2017.09.13
Phys 2016;96:296-303.

70. Peng P, Hyder O, Firoozmand A, et al. Impact of sarcopenia on outcomes following resection of pancreatic adenocarcinoma. J Gastrointest Surg 2012;16:1478-86.

71. van Dijk DP, Bakens MJ, Coolsen MM, et al. Low skeletal muscle radiation attenuation and visceral adiposity are associated with overall survival and surgical site infections in patients with pancreatic cancer. J Cachexia Sarcopenia Muscle 2017;8:317-26.

72. Mellon EA, Strom TJ, Hoffe SE, et al. Favorable perioperative outcomes after resection of borderline resectable pancreatic cancer treated with neoadjuvant stereotactic radiation and chemotherapy compared with upfront pancreatectomy for resectable cancer. J Gastrointest Oncol 2016;7:547-55.

73. Chuong MD, Frakes JM, Figura N, et al. Histopathologic tumor response after induction chemotherapy and stereotactic body radiation therapy for borderline resectable pancreatic cancer. J Gastrointest Oncol 2016;7:221-7.

74. Hong JC, Czito BG, Willett CG, et al. A current perspective on stereotactic body radiation therapy for pancreatic cancer. Onco Targets Ther 2016;9:6733-9.

75. Roeder F. Neoadjuvant radiotherapeutic strategies in pancreatic cancer. World J Gastrointest Oncol 2016;8:186-97. 


\section{Supplementary}

Table S1 Radiation toxicities

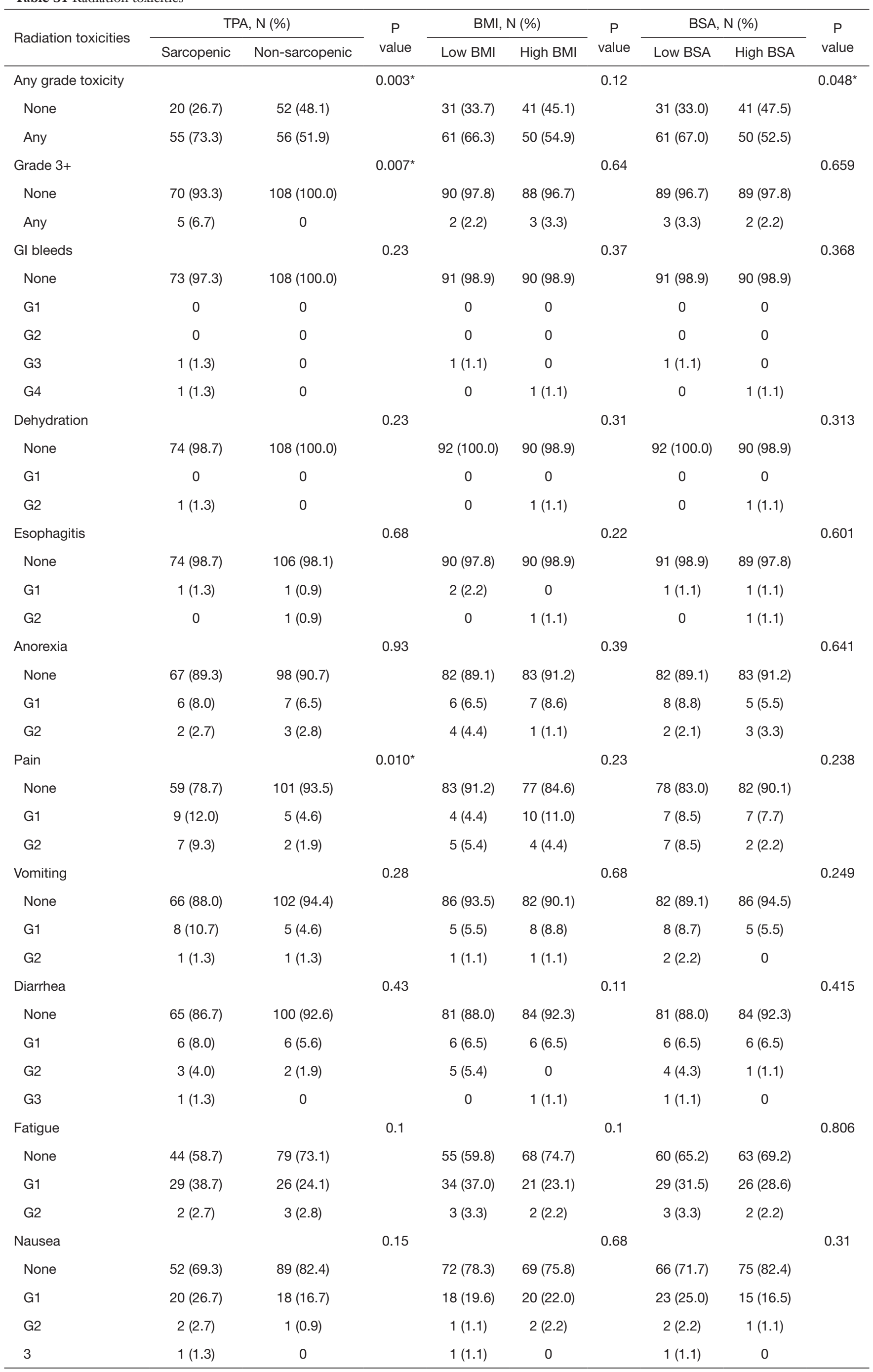

*, denotes significance at $\mathrm{P}<0.05$. TPA, total psoas area; BMI, body mass index; BSA, body surface area. 